\title{
Current advances in the application of proteomics in apoptosis research
}

\author{
WANG LiShun \& CHEN GuoQiang ${ }^{*}$ \\ Department of Pathophysiology, Key Laboratory of Cell Differentiation and Apoptosis of Ministry of Education of China; \\ E-Institutes of Shanghai Municipal Education Commission Chemical Biology Division, \\ Shanghai Jiao Tong University School of Medicine, Shanghai 200025, China
}

Received May 13, 2010; accepted May 26, 2010

\begin{abstract}
Apoptosis, or programmed cell death, is a complex, genetically-determined process involved in the development and maintenance of homeostasis in multicellular organisms. Dysregulation of apoptosis has been implicated in a number of diseases, including cancer and autoimmune disease. Thus, the investigation of apoptotic regulation has evoked considerable interest. Many apoptotic proteins have been shown to be post-translationally modulated, such as by protein cleavage, translocation, protein-protein interaction, and various post-translational modifications, which fall precisely within the range of proteomic analysis. Recently, contemporary proteomic technologies have achieved significant advances and have accelerated research in functional and chemical proteomics, which have been applied to the field of apoptosis research and have the potential to be a driving force for the field. This review highlights some of the major achievements in the application of proteomics in apoptosis research and discusses new directions and challenges for the near future.
\end{abstract}

apoptosis, proteomics, small compound, oncogene, tumor suppressor

Citation: Wang L S, Chen G Q. Current advances in the application of proteomics in apoptosis research. Sci China Life Sci, 2011, 54: 209-219, doi: $10.1007 / \mathrm{s} 11427-010-4123-0$

Most scientific advances benefit from the cross-talk among different strands of research and are fueled by new technologies. Our knowledge of apoptosis, one of the hottest areas in biomedical research, is also the outcome of interactions among many disciplines. Apoptosis is a genetically-determined cell suicide program that removes unwanted, redundant, or damaged cells, and it is required for the maintenance of tissue homeostasis in multicellular organisms [1]. Defects in the apoptotic program have been associated with many diseases, such as cancer, autoimmunity, and cardiac hypertrophy, whereas accelerated cell death is evident in degenerative diseases, immunodeficiency, and infertility [2]. Apoptosis is currently one of the most advanced fields of biological research, particularly regarding hypotheses and strategies linking molecular mechanisms to

*Corresponding author (email: chengq@ shsmu.edu.cn) disease phenotype. However, since apoptosis research began, many different research strategies and technologies from other subject areas have contributed to its development and have coalesced to deliver the present advanced knowledge of apoptosis.

Prior to the late 1960 s, research on cell death was mainly descriptive analyses of observations. With the development of light and electron microscopy, some features of cell death, such as DNA breakage, cell shrinkage, chromatin condensation, break-up of the cell and its engulfment, were recognized as being distinct from cell necrosis [1,3], and the term "apoptosis" was coined in 1972 by Kerr et al. [4]. In 1977, a cell lineage study based on the model organism Caenorhabditis elegans demonstrated that 131 out of the 1090 somatic cells were fated to undergo apoptosis during the development of $C$. elegans [5,6], paving the way for a genetic characterization of apoptosis. The central genetic compo- 
nents of the apoptosis machinery in C. elegans were revealed in the 1980s and 1990s, inspiring the discovery of their homologous proteins in humans in the 1990s [7]. In the early 1980s, research on gene translocations and oncogenic fusion proteins in leukemogenesis had become a very hot field and significant contributions were made to the investigation of apoptosis. Notably, $\mathrm{Bcl}-2$ was cloned from follicular lymphoma [8]. Initially, it was assumed to be similar to other oncogenes, such as $A b l$ and $c-M y c$, but it was finally found to cause cancer by preventing apoptosis rather than stimulating proliferation. Bcl-2 became the first identified component in apoptosis. Subsequently, other family members of $B c l-2$ were identified and the $B c l-2$ family is now known as one of the most important regulators of apoptosis [9]. The identification of components in the mitochondrial pathway is mainly based on biochemical separation strategies by the group of Wang [10]. The extrinsic apoptotic pathway was revealed by analysis of the Fas related protein complex $[7,11,12]$, while the link between apoptosis and immunological CTL killing led to the identification of granzyme B, a serine protease with a similar substrate specificity to the caspases, and revealed a type of non-cell autonomous apoptosis [3,13,14]. Examining the history of apoptosis research, it is clear that its development was fueled by various technologies and subjects, including microscopy, biochemistry, molecular biology, model organisms, as well as genetics, cancer biology, and immunology. Therefore, the role of proteomics in apoptosis research needs consideration

Apoptosis, a basic activity in cell biology, is under complicated and dedicated regulation [15]. In fact, an ever increasing number of genes are being revealed as involved in apoptotic events. In the pre-genomics era, genes/proteins would usually be selected manually to investigate their respective roles in a given apoptotic process. However, analysis using post-genomics tools might reveal sets of genes/ proteins that are up- or downregulated in a certain apoptotic event, allowing easier identification of novel molecules involved in the process. Microarray analysis of the genomic response to apoptosis induction is now well-established for gene screening; alterations in mRNA have been exhaustively described and many new genes were identified to be involved in apoptosis [16,17]. However, during cytotoxic stress after anticancer treatment, for example, protein translation is suppressed and post-translational modifications make the analysis of gene products even more complicated. It is unclear to what extent the genomic response is translated into alterations at protein levels [18]. The recent progress in proteomic technology, instrumentation for parallel quantitative proteome analysis of all proteins expressed from a genome, and for the identification of post-translational modifications has triggered a surge of chemical and functional proteomics studies dissecting the molecular mechanisms that regulate the apoptotic signal transduction pathways [19-23]. Despite the challenges of sensitivity and dynamic range, tremendous progress has been made in the field. This review summarizes the main progress in this field, including chemical compound-induced apoptosis, the proteomic investigation of apoptosis regulation, and caspase substrate identification. We also discuss the main challenges in this field and some possible future directions.

\section{Chemical compound-induced apoptosis}

Many small chemical compounds, including chemotherapeutic agents and some pollutants, are known to induce apoptosis. It is believed these chemical compounds induce mitochondria to undergo outer membrane permeabilization and to release cytochrome $\mathrm{C}$. The latter facilitates the interaction of Apaf- 1 with upstream caspase- 9 and initiates a downstream caspase cascade, especially the activation of caspase-3, eventually leading to apoptosis [24]. However, the initial signaling triggering apoptosis, as well as the cellular context, may vary significantly, thus the mechanisms and signaling pathways usually demonstrate apoptotic stimuli and cellular context specificities [24]. Determining the detailed molecular pathways by which chemical compounds induce apoptosis will provide valuable information for the further development of novel drug leads and toxicity evaluations. Proteomic approaches, combined with other biochemical methods, can reconstruct regulatory networks, signaling cascades, and metabolic pathways upon chemical stimulus, which demonstrates the value of proteomic platforms for identifying drug targets, understanding the exact mechanisms behind the induction of cell death in response to these agents, and in the evaluations of toxicity and side effects of these compounds [20,23,25].

Apoptotic impotence is frequently involved in carcinogenesis and resistance to cancer treatment, and thus inducing apoptosis is one of the main strategies used for cancer chemotherapy [2,26]. However, the network of apoptosis regulation is highly complex and one single drug usually does not work well. The development of novel anticancer chemotherapeutic drugs whose mechanisms of action are different from established drugs is needed for the treatment of cancer, particularly in those patients showing resistance to standard treatment $[27,28]$. However, the development of therapeutics from inception to marketing is a costly and time-consuming path with high attrition rates. The identification of drug targets involved in compound-induced apoptosis is an important factor for rational application and a major rate-limiting step in anticancer drug development, for which proteomic analysis may provide valuable information. For example, arsenic trioxide exerts its therapeutic effect for patients relapsed after ATRA treatment by promoting cell differentiation and apoptosis [29], but the drug target involved is not clear. Very recently, Zhang et al. [30] used biotin-linked arsenic trioxide to purify interacting proteins; 
they found that arsenic binds directly to the cysteine residues in the zinc finger motifs located within the RBCC domain of PML-RARalpha and PML. Arsenic binding induces PML oligomerization, which increases its interaction with the small ubiquitin-like protein modifier (SUMO)-conjugating enzyme UBC9, resulting in enhanced SUMOylation and degradation. Arsenic and ATRA bind to PML at different sites and this information could be useful for their rational application. Another example is Ganoderic acid D (GAD), one of the major components in Ganoderma triterpenes, which can inhibit the growth of numerous cancer cell lines and were thought to be the basis of their anticancer effects [31]. Yue et al. [31] used two-dimensional gel electrophoresis to analyze the proteins with changes in expression level after GAD treatment, and 21 proteins, including 14-3-3, were identified. Furthermore, GAD was found to possess the ability to bind six isoforms of the 14-3-3 protein family and other proteins by the drug target searching program analysis. The direct binding affinity of GAD towards 14-3-3 zeta was confirmed in vitro using surface plasmon resonance biosensor analysis. In addition, 14 of the 21 proteins whose expression changed in the proteomics analysis were closely related in a protein-protein interaction network identified in the intensive study. In this way, the possible network associated with GAD target-related proteins was constructed [32].

Meanwhile, the prompt and accurate interpretation of the molecular mechanisms for a potential drug function will accelerate the process and significantly decrease the cost involved in drug development. Proteomics was found to be very useful in uncovering the mechanisms of novel reagents. For example, gold(III) complexes have the potential to become a new class of anticancer drugs with higher cytotoxicity and fewer side effects than existing metal anticancer drugs, such as cisplatin [33], and their mechanism of action has been eagerly sought. Wang et al. [34] performed functional proteomic studies on the apoptotic HONE1 cells induced by gold(III) porphyrin 1a and revealed alterations in the levels of proteins involved in cellular redox balance. Further study showed that ROS played a part in gold(III) porphyrin 1a-induced apoptosis by regulating DeltaPsi(m). These results from proteomics analysis support a role for gold(III) porphyrin 1a as a promising novel anticancer drug directed toward the mitochondria. Proteomics has a limitation for detection and sensitivity for low abundance proteins, and can only reveal the most significant changes in high abundance proteins, which may demonstrate only some of the important parts of the drug effects.

Drug resistance is one of the major problems for successful chemotherapy for cancer and other diseases. Many molecular mechanisms have been revealed to be responsible for drug resistance, whereas others will surely be discovered. Drug resistance might be the result of the activation of different mechanisms and determining the exact mechanism activated in a particular case is a difficult task [35]. Pro- teomics could be applied to the investigation of the drug resistance mechanisms through the identification of entire sets of proteins that are up- or downregulated in chemoresistant cell lines compared with their parental lines. For example, the therapeutic mainstay against the protozoan parasite Leishmania is still based on the antiquated pentavalent antimonials $(\mathrm{Sb}(\mathrm{V}))$, which induce apoptosis in the parasite, but resistance is increasing in several parts of the world [36]. To reveal the mechanism of this resistance, Vergnes et al. [37] performed a comparative analysis of a genetically related pair of $\mathrm{Sb}(\mathrm{V})$-sensitive and -resistant Leishmania donovani strains isolated from kala-azar patients. They identified that heat shock protein HSP83 and the small kinetoplastid calpain-related protein (SKCRP14.1) were differentially expressed. They found that HSP83 increased drug resistance and reduced drug-mediated programmed cell death (PCD) activation by interfering with the mitochondrial membrane potential, whereas SKCRP14.1 promoted antimonial-induced PCD but protected against miltefosine-induced PCD.

On the other hand, drugs can have side effects. Understanding the molecular mechanisms involved in the side effects will aid the reasonable application of the drug and help develop methods for avoiding its side effects. For example, mycophenolic acid (MPA) has been widely used as an immunosuppressive drug after transplantation [38]. MPA-induced beta-cell toxicity is an important factor for islet graft function. The signal transduction mechanism underlying this process has not been fully explored. Using a proteomics approach, Park et al. [39] examined protein expression patterns in MPA-treated apoptotic RIN-5 cells and found a decrease in the expression of RhoGDI-alpha. Over-expression of RhoGDI-alpha increased cell viability and decreased activated JNK expression following exposure to MPA, whereas knockdown of RhoGDI-alpha enhanced MPA-induced cell death and increased the activation of JNK. MPA-induced cell death was prevented by a JNK inhibitor. The proteomic analysis indicated that MPA induces significant apoptosis in insulin-secreting cells via the downregulation of RhoGDI-alpha linked with increased JNK expression. This RhoGDI-alpha/JNK pathway might be the focus of therapeutic target for the prevention of MPA-induced islet apoptosis.

Pollutants in the environment have increased in the past years, coincident with economic development. Some environmental contaminants demonstrated toxicity on organisms via apoptosis induction. Proteomic analysis of these compounds could provide useful information for human health protection. The environmental contaminant 2,3,7,8-tetrachlorodibenzo-p-dioxin (TCDD) has been shown to cause a wide variety of toxic effects and is regarded as the most potent hepatocarcinogen in experimental animals [40]. For a comprehensive survey of the impact of TCDD on the proteome of hepatic cells, Sarioglu et al. [41] performed a high resolution $2 \mathrm{D}$ gel electrophoresis study on the rat hepatoma 
cell line 5L exposed to TCDD. A particularly intriguing finding was that the upregulation of the mitochondrial outer membrane pore protein, voltage-dependent anion channelselective protein 2 (VDAC2), depended on the presence of a functional aryl hydrocarbon receptor. In view of the central role of VDAC2 as an inhibitor of the activation of the proapoptotic protein $\mathrm{BAK}$, and the mitochondrial apoptotic pathway, their data point to a hitherto unrecognized mechanism by which TCDD might affect cellular homeostasis and survival. Another example is 1,2-dichlorovinyl-L-cysteine (DCVC), which belongs to the group of nephrotoxic halogenated alkene S-cysteine conjugates and is specifically taken up by renal proximal tubular cells both in vitro and in vivo where it is bio-activated by the renal cysteine conjugate-lyase activity, resulting in the formation of reactive intermediates that cause cell injury [42]. De Graauw et al. [43] used 2D-DIGE to determine early changes in the onset of apoptosis of renal epithelial cells induced by DCVC. They identified a significant change in HSP27, which demonstrated a pI shift in association with an altered phosphorylation status. They also found that the inhibition of $\mathrm{p} 38$ by SB203580 reduced HSP27 phosphorylation and accelerated reorganization of focal adhesions, cell detachment, and apoptosis. Overexpression of a phosphorylation-defective mutant HSP27 had a dominant-negative effect and accelerated the DCVC-induced changes in focal adhesions as well as the onset of apoptosis. Their proteomic analysis indicated that phosphorylation of HSP27 via p38 activation might be a possible means to maintain cell adhesion, and thus suppress renal epithelial cell apoptosis by DCVC.

While some small molecular compounds may not be drug leads, they might have special activities in biological systems that could lead to the discovery of unknown mechanisms and pathways involved in these complex biological processes. For example, apoptosis triggered by endoplasmic reticulum (ER) stress has been implicated in many diseases, but its cellular regulation remains poorly understood [44]. Salubrinal is a small molecule that protects cells from ER stress-induced apoptosis by selectively activating a subset of endogenous ER stress-signaling events [45]. Boyce et al. [46] used salubrinal as a probe in a DIGE-based proteomic approach to discover new information about the endogenous cellular response to ER stress. They found that one set of protein spots shifted toward the anode of the IEF dimension in response to salubrinal treatment. These spots were identified as eEF-2 by mass spectrometry. A shift toward the anode in the IEF dimension is consistent with a phosphorylation event and they subsequently identified the phosphorylation site (residues 55-59 of rat eEF-2). Using this information, they tracked the upstream protein kinase event and identified eEF-2K as a hitherto unknown regulator of ER stress-induced apoptosis.

We found that a new camptothecine derivate, NSC606985, could induce AML cell apoptosis via proteolytic activation of protein kinase delta (PKC $\delta$ ) at nanomole concentrations [47].
NSC606985 also demonstrated therapeutic effects on an AML leukemic mouse [48]. We then performed dynamic proteomic analysis of leukemic NB4 cells with NSC606985 as an apoptosis-inducing probe and identified a large set of proteins that were deregulated in apoptosis [18]. In combination with bioinformatics, a hypothetical network of these deregulated proteins was constructed and provided clues to new apoptosis mechanisms [27]. Based on these clues, we found that the acidic leucine-rich nuclear phosphoprotein 32B (ANP32B) was a substrate of caspase-3 and that the knocking down of ANP32B sensitized the cells to apoptotic stimuli [49]. More intriguingly, further analysis showed that the ANP32B caspase-3 cleavage fragment might have important roles in apoptotic regulation (data not shown). In addition, hnRNPK was also found to decrease via proteasome degradation during apoptosis, and its knockdown also contributed to apoptosis induction [50]. In addition, we found that dihydrofolate reductase (DHFR) is translocated from the cytosol to the nucleus during the apoptosis [51]. Recent data from our laboratory supported the view that this translocation was required for apoptosis induction under certain conditions such as ER stress. Among these findings, most of the protein deregulations occurred later in apoptosis. With the advantage of easy control of apoptosis induction via dosage and time modification for chemical compound treatment, we found that the expression of N-myc downstream-regulated gene 1 (NDRG1) decreased prior to the cleavage of PKC $\delta$ in apoptosis induced by NSC606985. In addition, NDRG1 downregulation was found to sensitize the leukemic cells to apoptosis induction by promoting the proteolytic activation of PKC $\delta$ [52].

\section{Tumor suppressors and oncogenes}

The apoptosis process is finely regulated by a series of modulators. For example, tumor suppressors, such as wildtype $p 53$ and Bax, and oncogenes such as mutant P53, Bcl-2, and bcl-XL have been proposed to have crucial roles in modifying the susceptibility of normal and cancer cells to the induction of apoptosis [26,53]. Research into the modulation of apoptosis-regulating genes has thus evoked great interest and proteomics has been frequently used as an effective research strategy.

Proapoptotic B-cell lymphoma-2 (Bcl-2) family members are the requisite arbiters of the mitochondrial apoptotic pathway [54]. The Bcl-2 family is conserved through evolution and consists of both proapoptotic and anti-apoptotic members. The Bcl-2 family is divided into three sub-groups. The first group includes the pro-apoptotic members BAX and BAK, which constitute a requisite gateway to the mitochondrial pathway of apoptosis, and their allosteric activation results in the permeabilization of the outer mitochondrial membrane and the release of cytochrome $\mathrm{C}$. The second group is the $\mathrm{BH} 3$-only molecules, including $\mathrm{BAD}, \mathrm{BID}$, 
and others, which can result in the activation of BAX and BAK or can be sequestered by the anti-apoptotic Bcl-2 and $\mathrm{Bcl}-\mathrm{XL}$. The third group is $\mathrm{Bcl}-2, \mathrm{Bcl}-\mathrm{XL}$, and other anti-apoptotic members in the family. The fine balance between the expression levels of pro- versus antiapoptotic Bcl-2 family members, as well as interactions among them, determines whether the cell lives or dies, and this process is regulated at the transcriptional or post-translational level. However, the detailed mechanisms of the interactions of Bcl-2 family and the activation of BAX and BAK are still not clear, and constitute the Holy Grail of apoptosis research [9]. To purify the interacting proteins of BAX, Kerr et al. [55] used the native 21 amino-acid $\mathrm{C}$ terminus of Bax, which does not result in mitochondrial translocation on its own, and its S184V mutant peptide, which artificially mimics the correct conformation for targeting Bax to the mitochondria. Using columns of biotinylated peptides coupled to agarose beads, affinity chromatography was performed using SH-SY5Y cell lysates and bound proteins were separated by SDS-PAGE followed by mass spectrometry identification. They found that the molecular chaperone nucleophosmin was a novel Bax-binding protein. Specific RNAimediated knockdown of nucleophosmin expression attenuated apoptosis. Thus, they elucidated a novel molecular mechanism whereby Bax becomes activated and translocates to the mitochondria to orchestrate mitochondrial dysfunction and apoptotic cell death. On the other hand, another proteomic investigation found that Bax could also be transcriptionally regulated by knocking down CTCF, which is highly expressed in breast cancer cell lines and tumors compared with breast cell lines with finite life span and normal breast tissues. This analysis supports the hypothesis that the upregulation of CTCF might be linked to the resistance of breast cancer cells to apoptosis [56]. Notably, BAK and BAX are usually thought to act as requisite arbiters of mitochondria. However, Hwang et al. [57] used a SILAC (stable isotope labeling by amino acids in cell culture)based unbiased quantitative proteomic analysis of nuclear fraction of Jurkat cells during apoptosis induced by anti-Fas antibody and found that the mitochondrial protein BAK was dynamically recruited into nuclear invaginations during apoptosis. This might indicate that BAK has roles in the nucleus other than its known roles in mitochondria. In a gel filtration chromatography-based proteomic analysis, Danial et al. [58] found that the BH-3-only pro-apoptotic BAD resides in a functional holoenzyme complex together with glucokinase and is required to assemble the complex in liver mitochondria. Notably, in BAD-deficient hepatocytes that lack this complex, mitochondria-based glucokinase activity, and mitochondrial respiration in response to glucose, diminished. Glucose deprivation results in dephosphorylation and activation of BAD, and induces BAD-dependent cell death. This proteomics-based protein complex analysis indicates an unanticipated role for BAD in integrating pathways of glucose metabolism and apoptosis. Liu et al. [59] identified JAB1 as a novel BclGs-specific binding protein through a yeast two-hybrid screening in a human testis cDNA library. The N-terminal region of BclGs was required for the interaction. JAB1 could compete with Bcl-XL/Bcl-2 to bind to BclGs and thus promote apoptosis. Cimmino et al. [60] found that cells overexpressing "wild-type" human isoaspartyl protein carboxyl-O-methyltransferase (PCMT) were resistant to apoptosis, whereas overexpression of antisense PCMT induced high sensitivity to apoptosis, even at low $\mathrm{H}_{2} \mathrm{O}_{2}$ concentrations. By incubating the purified human recombinant PCMT with cell lysate, they identified that Bcl-xL was a specific methyl-accepting substrate of PCMT and thus lost its anti-apoptotic activity for the methylation modification. In a quantitative proteomic analysis for Myc-induced apoptosis using an isotope-coded affinity tag, the expression of $\mathrm{Bcl}-2$ and $\mathrm{Bcl}-\mathrm{xL}$ was found to be suppressed, which indicated that the increase of Bcl-2 and Bcl-xL in tumor cells might be one of the means to escape Myc-induced apoptosis [61]. These proteomics analyses provided valuable information on the molecular mechanisms of Bcl-2 family in apoptosis regulation.

As an important tumor suppressor protein, p53 is often referred to as the "guardian of the genome" [53]. p53 responds to DNA-damage or checkpoint failure and gives rise to a series of anti-proliferative responses, including apoptosis induction through transcription dependent or independent mechanisms, while disruption of this route can promote tumor progression and chemoresistance. For example, approximately $50 \%$ of sporadic human tumors harbor somatic mutations in the p53 gene locus. Therefore, to ensure normal cell growth, p53 levels and activity are tightly regulated. Post-translational modification following exposure to a variety of stresses is one of the major phenomena of p53. However, there have been conflicting reports on whether p53 post-translational modifications, such as phosphorylation or acetylation, are essential or only play a subtle, fine-tuning role in the p53 response. In a cleavable ICAT quantitative proteomics analysis of thymocytes from p53(K317R) knock-in mice treated by ionizing radiation, 46 proteins were found to be differentially affected [62]. Pathway analysis of these proteins suggested an increase in p53 activity in the $\mathrm{p} 53(\mathrm{~K} 317 \mathrm{R})$ thymocytes as well as a decrease in tumor necrosis factor alpha signaling. These results suggest that the acetylation of Lys-317 modulates the functions of p53 and influences the cross-talk between the DNA damage response and other signaling pathways [62]. A milestone work was recently reported by Tang et al. [63]. They identified all major acetylation sites of p53 based on a proteomic analysis. They also found that the loss of acetylation completely abolished p53-dependent growth arrest and apoptosis. Acetylation of p53 abrogated Mdm2-mediated repression by blocking the recruitment of $\mathrm{Mdm} 2$ to $\mathrm{p} 53$ responsive promoters, which leads to p53 activation independent of its phosphorylation status. This study demonstrated p53 acetylation as an indispensable event for desta- 
bilizing the p53-Mdm2 interaction and enabling the p53-mediated stress response.

The tumor suppressor p53 executes its function partially by transcriptional regulation of a set of target genes. Clearly, identification of new p53 targets is important to achieve a better understanding of the p53 tumor suppressor pathway. Gu et al. [64] compared the proteome of a human colorectal cancer cell DLD-1 transfected with inducible p53 with that of the control cell line using amino acid-coded mass tagging-assisted mass spectrometry. They identified a large dataset of proteins that are upregulated at the execution stage of p53-mediated apoptosis. Meanwhile, using ICAT labeling and high throughput mass spectrometry, Johnson et al. [65] identified in vivo quantitative changes in wild-type p53 mouse cortical neurons undergoing camptothecine DNA damage-induced death and found a large set of changed proteins. Using a 2DE-based quantitative proteomic method, Rahman-Roblick et al. [66] compared the total

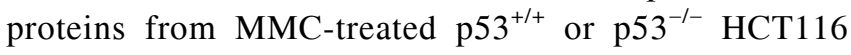
cells and found many deregulated proteins. In addition to transcriptional regulation, it is conceivable that p53 regulates targets at the post-translational level. In fact, RahmanRoblick et al. [66] demonstrated that both transcriptiondependent and transcription-independent regulation were among the identified targets in a previous study. Furthermore, Rahman-Roblick et al. [67] examined p53-dependent phosphorylation using 2DE and staining with the fluorescent phosphoprotein dye Pro-Q Diamond and reported a subset of putative protein phosphorylation events. Orre et al. [68] showed that the phosphorylation of S100A6 is dependent on p53 using SELDI-TOF based top-down mass spectrometry. Thus, proteomic analysis could increase the understanding of the mechanisms of p53 regulation and biological function, which then provide the basis for new drug designs that could eventually lead to therapeutics to reactivate $\mathrm{p} 53$ in cancers.

The transmission of apoptotic signals from the plasma membrane to the nucleus involves a number of different pathways and protein modifications, primarily in the form of phosphorylation, which lead to the activation of a series of protein kinases. Activation of specific protein kinases can promote cell death in some circumstances, while others protect the cell against apoptosis [69]. Thus, the discovery of protein kinase substrates would achieve a better understanding of apoptotic signaling. Protein-protein interactions between kinases and substrates are required for the phosphorylation reaction and thus protein-protein interaction studies are an effective strategy to identify the substrates as well as the regulators of the kinases. For example, Galietta et al. [70] identified several RNA/DNA-binding proteins, including protein-associated splicing factor (PSF), that coimmunoprecipitated with NPM/ALK. PSF was revealed as a substrate of ALK by further analysis, and ALK fusion proteins could induce phosphorylation and delocalization of PSF from the nucleus to the cytoplasm in NPM/ALK(+) cells. PSF phosphorylation decreased the PSF-mediated suppression of GAGE6 expression and possibly contributed to the pathogenesis of lymphoma. In an unbiased proteomic screening using yeast two-hybrid assays to identify integrinlinked kinase (ILK) interactors, rictor was revealed as an ILK-binding protein. Single depletion of rictor decreased ILK-related Akt Ser (473) phosphorylation and depletion of ILK and rictor in breast and prostate cancer cell lines resulted in the inhibition of Akt Ser (473) phosphorylation and induction of apoptosis. These data show that rictor regulates the ability of ILK to promote Akt phosphorylation and cancer cell survival [71]. With the development of strategies and technologies for the enrichment of phosphorylated protein/peptides and the improvement in sensitivity and throughput of MS analysis, phosphorylation profiling analysis has become an alternative option to identify the protein substrates of kinases. To better understand the signaling properties of oncogenic FGFR3, Kang et al. [72] performed phospho-proteomic studies and identified many potential downstream signaling effectors that are tyrosine phosphorylated in hematopoietic cells expressing constitutively activated leukemogenic FGFR3 mutants. Furthermore, they found that FGFR3 directly phosphorylates the serine/ threonine kinase p90RSK2 at Y529. This phosphorylation consequently regulates RSK2 activation by facilitating inactive ERK binding to RSK2, which is required for ERK-dependent phosphorylation and activation of RSK2, which, in turn, mediates hematopoietic transformation.

Intriguingly, some canonical kinases might have an important role via kinase-independent pathways. For example, the serine/threonine kinase Raf acts as an important factor in the ras pathway [73], but some studies found that ablation of the Raf-1 gene causes widespread apoptosis and embryonic lethality, despite normal regulation of ERK through B-Raf [74]. Similarly, Raf-1 deficient fibroblasts are hypersensitive to apoptosis that is induced by selected stimuli, including serum withdrawal and stimulation of the death receptor Fas. Knock-in of Raf-1 in a Raf-1 mutant that cannot be activated fully rescued the phenotype. The ablation of the protein kinase Raf-1 renders cells hypersensitive to apoptosis, despite normal regulation of extracellular signalregulated kinases, suggesting that apoptosis protection is mediated by an unknown pathway. To search for new partners in Raf-1 signaling, O'Neill et al. [75] immunopurified proteins associated with flag-tagged Raf-1 that were expressed in COS-1 cells, and identified that Raf- 1 counteracts apoptosis by suppressing the activation of mammalian sterile 20-like kinase (MST2). Raf-1 prevents dimerization and phosphorylation of the activation loop of MST2 independently of its protein kinase activity. Depletion of Raf-1 from mouse or human cells led to MST2 activation and apoptosis. Depletion of MST2 from Raf- $1^{-1-}$ mouse or human cells abrogated sensitivity to apoptosis, whereas overexpression of MST2 induced apoptosis. Furthermore, they immunoprecipitated MST2 and identified LATS1 as a 
MST2 binding partner. They found that the tumor suppressor gene RASSF1A disrupted the inhibitory Raf1-MST2 complex and enhanced the MST2 interaction with its substrate, LATS1. Subsequently, RASSF1A-activated LATS1 phosphorylates and releases the transcriptional regulator YAP1, allowing YAP1 to translocate to the nucleus and associate with $\mathrm{p} 73$, resulting in transcription of the proapoptotic target gene, puma [76].

\section{Caspases and other protease substrates}

Since human caspase-1 was identified as the $C$. elegans homolog of CED-3, the caspase family has been the subject of intense research because of their distinct physiological functions, substrates, activation mechanisms, and signal transduction pathways. This research has made a large contribution to the understanding of the molecular mechanisms of apoptosis as well as demonstrating the involvement of caspases in the pathogenesis of certain diseases [7,24]. Notably, as aspartic acid-specific proteases, caspases have crucial roles in apoptosis via specific proteolytic cleavage of protein substrates. It is probable that the cleavage of some of these substrate proteins simply represent "innocent bystanders" that are degraded following the death program. However, a few of these protein cleavages, such as caspases, $\mathrm{RB}$, IAP, and PARP, have been rigorously established as biologically relevant steps in protein activation and translocation, and have important function in the signature events in apoptosis, such as membrane phosphatidylserine externalization, cellular retraction, chromatin condensation, and apoptotic body production [77]. In fact, in our laboratory, we have identified that the leukemic fusion protein AMLETO, and ANP32B, are substrates of caspase- 3 and their products play important roles in apoptosis regulation [78].

In addition, substrate identification aids in revealing enzymatic functions. For example, members of the caspase family of proteases have crucial roles in coordinating apoptotic events and it was assumed that the major executioner caspases, caspase- 3 and caspase-7, exhibit functionally redundant roles within the cell death machinery because they show similar activity toward certain synthetic peptide substrates [79]. However, the distinct phenotypes of mice deficient in these caspases make this assumption controversial [80]. Using cell-free caspase-3-depleted Jurkat extracts and 2DE analysis, Walsh et al. [81] showed that caspase- 3 and caspase-7 exhibit differential activity toward multiple substrate proteins. The removal of caspase- 3 abolished the vast majority of caspase-dependent proteolytic changes evident in this system, with few proteins undergoing proteolysis in the caspase-3-depeleted extract. This suggested that caspase- 3 is more promiscuous than caspase7 and appears to be the major executioner caspase during the demolition phase of apoptosis. Thus, the recognition of caspase substrates during the demolition phase of apoptosis was one of the focuses of apoptosis research. In the past decade, the list of proteins that are reported to be cleaved by caspases in vivo and in vitro has grown rapidly and several hundred substrates of apoptosis-associated caspases have been reported [77,82-84]. These substrates usually share relatively common sequences (for example, tetrapeptide sites after a highly conserved aspartic acid residue) within proteins and it was thought that there are likely to be hundreds more intracellular proteins yet to be discovered that match the consensus substrate specificity of caspases. For example, bioinformatics has been applied to predict substrates in the genome and has provided many putative substrates and cleavage sites [85]. However, these putative substrates remain to be experimentally verified. Therefore, there is considerable interest in the development of methodologies that would allow for a comprehensive characterization of the proteolytic pathways in apoptotic systems. Innovative proteomics-based methods and high-throughput protein separation and identification have significantly influenced the search for caspase substrates on a global scale.

Dix et al. [86] set up a robust and conventional gel-based proteomic platform that enabled direct visualization of the topography and magnitude of proteolytic events on a global scale. Protein samples were extracted from control native and apoptotic cells and separated by SDS-PAGE. Full-length proteins will be present in the control native lane while the fragments of the native proteins after cleavage will be present in the apoptotic cell lane with a lower molecular weight. To identify the proteins, the SDS-PAGE gel was sliced into strips, followed by trypsin digestion and LC-MS/MS analysis. The presence of the same peptides at different molecular weights thus provided information about the identity of the cleaved proteins. They identified 91 characterized caspase substrates as well as 170 additional proteins previously not known to be cleaved during apoptosis. Surprisingly, they found that the vast majority of proteolyzed proteins, regardless of the extent of cleavage, yielded persistent fragments that corresponded to discrete protein domains, which suggested that the generation of active effector proteins might be a principal function of apoptotic proteolytic cascades [86]. Mahrus et al. [87] presented an intriguing approach to the problem of how to specifically label the $\mathrm{N}$ termini of proteins to allow for the enrichment of corresponding $\mathrm{N}$-terminal peptides and identification of the locations of protease cleavage sites. They used subtiligase to tag free $\mathrm{N}$ termini with a biotin peptide after apoptosis induction, followed by LC-MS/MS analysis and database searching. They have sequenced 333 caspase-like cleavage sites distributed among 292 protein substrates. They also found that caspase cleavage sites often appear in surface-accessible loops, and a disproportionate number of caspase substrates physically interact, suggesting that these dimeric proteases target protein complexes and networks to elicit apoptosis. With the rapid advance of quantitative proteomic technology, including the SILAC and ITRAQ (isobaric tag for relative 
and absolute quantitation) [88], more and more powerful methods could be developed and applied to discover more novel caspase substrates and cleavage sites. Accordingly, the proteins cleaved by caspases will form an even longer list. It is necessary to summarize these proteins as a database and even identify the subset of these proteins that have functional significance for the process of apoptosis and/or inflammation. Luthi et al. have compiled a comprehensive list of caspase substrates (The Casbah; www.casbah.ie), which contains information pertaining to most of the currently known caspase substrates [83].

It has been long supposed that several other proteases are engaged in cleavage activity during the apoptotic process, which has been demonstrated by several investigations, including proteomic efforts. Van et al. [89] used fractional diagonal chromatography sorting of protein amino-terminal peptides coupled to oxygen-16 or oxygen-18 differential labeling to discover the protein cleavage in anti-Fas-treated apoptotic human Jurkat $\mathrm{T}$ lymphocytes. They located 93 cleavage sites in 71 proteins in anti-Fas-treated apoptotic human Jurkat $\mathrm{T}$ lymphocytes. As expected, most cleavages were at caspase consensus sites. However, 35 other cleavages occurred C-terminally to nonaspartate residues, mainly involving either basic or bulky hydrophobic amino acids, suggesting activation of other proteases. Similarly, Impens et al. [90] analyzed the involvement of proteases during taxol-mediated cell death of human A549 non-small-cell lung carcinoma cells using a proteomic approach. They revealed 27 protease-mediated cleavages, which included cleavage sites $\mathrm{C}$-terminal to aspartic acid and sites $\mathrm{C}$-terminal to non-Asp residues, as the result of caspase and non-caspase protease activities, respectively.

Among these non-caspase proteases involved in apoptotic process, Granzyme B, a major cytotoxic T lymphocyte/natural killer (CTL/NK) granule protease, was the most widely investigated [13]. Granzyme B was known to activate members of the caspase family of cysteine proteases through processing of caspase zymogens, and can thus initiate apoptosis under certain stimuli [14]. However, it is supposed that more proteins could be the potential substrate of Granzyme B during apoptosis. To identify the specific protein substrates from the huge number of cleavages in the apoptotic products, Bredemeyer et al. [91] treated YAC-1 cells with granzyme B in the presence of caspase inhibitors and used DIGE (differential gel electrophoresis) and tandem MS to identify substrates of granzyme B. They found procaspase-3 and several other substrates of granzyme B. Alternatively, Adrain et al. [92] used a cell-free system and a 2DE-based proteomics approach to investigate molecular order and relative importance of caspase activation events that occur in target cells during granzyme B-initiated apoptosis. They examined the scale of granzyme B-initiated alterations to the proteome in the presence or absence of effector caspase- 3 or -7 . Their study indicated that granzyme B targets a highly restricted range of substrates and orches- trates cellular demolition largely through activation of caspase-3.

The mitochondrial serine protease high-temperature requirement protein A2 (HtrA2/Omi) is expressed from a nuclear gene as a proenzyme of $49 \mathrm{kD}$ with an N-terminal mitochondrial localization signal that mediates its translocation into the mitochondrial intermembrane space. HtrA2/ Omi is one of the factors that are released into the cytosol after apoptotic stimuli [93]. The proteolytic activity of HtrA2/Omi was found to have an important role in caspasedependent apoptosis and, more importantly, it is also required in a caspase-independent manner. However, except for IAP [94], very few targets of HtrA2/Omi were known. To identify apoptotic targets of HtrA2/Omi, Vande Walle et al. [95] purified recombinant HtrA2/Omi and its catalytically inactive S306A mutant. Lysates of human Jurkat $\mathrm{T}$ lymphocytes incubated with either wild-type recombinant HtrA2/Omi or the S306A mutant and subjected to mass spectrometry identification yielded $15 \mathrm{HtrA} 2 / \mathrm{Omi}$ substrates and 50 cleavage sites of potential physiological relevance. Several processing events were validated by incubating purified recombinant HtrA2/Omi with in vitro translated substrates or with Jurkat cell lysates. In addition, the set of cleavage sites was used to assess the protein substrate specificity of HtrA2/Omi. The results suggest that HtrA2/Omi has a rather narrow cleavage site preference and that cytoskeletal proteins are the prime targets of this protease.

\section{Summary}

This review summarizes the major advances in functional proteomics and chemical/drug proteomics in apoptosis research. With throughput and sensitivity that are unparalleled by traditional biochemical methods, proteomic strategies and technologies have demonstrated particular advantages in substrate identification for proteases and protein kinases which are critical for apoptosis and for other biological processes such as inflammation. Sensitivity and throughput are still being improved to accommodate the highly demanding nature of genome-wide identification of proteins. At present, only highly abundant proteins can be identified in whole cells or tissue lysate, and various protein isolation and purification strategies are needed to identify certain groups of proteins.

With the length limitation of this review, much interesting work was not included. For example, for the proteomic analysis of post-translational modifications in apoptosis research, we focused on phosphorylation because of its long research history and relatively mature technologies. Much excellent work will emerge as a result of the fast development of other PTM proteomic technologies, such as acetylation, SUMOylation, ubiquitination, glycosylation, and methylation. Furthermore, new technologies that investigate 
protein-protein interactions, small compound-protein interactions, and protein-DNA/RNA interactions will reveal even more important apoptosis regulators and mechanisms.

This work was supported in part by the Ministry of Science and Technology of China (Grant Nos. 2006CB910104, 2006AAZ105 and 2009CB918404), the National Natural Science Foundation of China (Grant Nos. 30630034, 90813034 and 81071668), the Shanghai Science and Technology Commission (Grant Nos. 08JC1413700 and 07QA14041) and the Shanghai Municipal Education Commission (Grant No. E09013).

1 Raff M C. Social controls on cell survival and cell death. Nature, 1992, 356: 397-400

2 Vermeulen K, Van Bockstaele D R, Berneman Z N. Apoptosis: Mechanisms and relevance in cancer. Ann Hematol, 2005, 84: 627-639

3 Vaux D L. Apoptosis timeline. Cell Death Differ, 2002, 9: 349-354

4 Kerr J F, Wyllie A H, Currie A R. Apoptosis: A basic biological phenomenon with wide-ranging implications in tissue kinetics. $\mathrm{Br} \mathrm{J}$ Cancer, 1972, 26: 239-257

5 Ellis H M, Horvitz H R. Genetic control of programmed cell death in the nematode C. elegans. Cell, 1986, 44: 817-829

6 Sulston J E, Horvitz H R. Post-embryonic cell lineages of the nematode, Caenorhabditis elegans. Dev Biol, 1977, 56: 110-156

7 Li J, Yuan J. Caspases in apoptosis and beyond. Oncogene, 2008, 27: 6194-6206

8 Vaux D L, Cory S, Adams J M. Bcl-2 gene promotes haemopoietic cell survival and cooperates with c-myc to immortalize pre-B cells. Nature, 1988, 335: 440-442

9 Youle R J, Strasser A. The Bcl-2 protein family: Opposing activities that mediate cell death. Nat Rev Mol Cell Biol, 2008, 9: 47-59

10 Liu X, Kim C N, Yang J, et al. Induction of apoptotic program in cell-free extracts: Requirement for dATP and cytochrome c. Cell, 1996, 86: 147-157

11 Micheau O, Tschopp J. Induction of TNF receptor I-mediated apoptosis via two sequential signaling complexes. Cell, 2003, 114: 181-190

12 Varfolomeev E E, Schuchmann M, Luria V, et al. Targeted disruption of the mouse Caspase 8 gene ablates cell death induction by the TNF receptors, Fas/Apo1, and DR3 and is lethal prenatally. Immunity, 1998, 9: 267-276

13 Trapani J A, Smyth M J. Functional significance of the perforin/granzyme cell death pathway. Nat Rev Immunol, 2002, 2: 735-747

14 Andrade F, Roy S, Nicholson D, et al. Granzyme B directly and efficiently cleaves several downstream caspase substrates: Implications for CTL-induced apoptosis. Immunity, 1998, 8: 451-460

15 Chowdhury I, Tharakan B, Bhat G K. Current concepts in apoptosis: The physiological suicide program revisited. Cell Mol Biol Lett, 2006, 11: 506-525

16 Hofmann W K, de Vos S, Tsukasaki K, et al. Altered apoptosis pathways in mantle cell lymphoma detected by oligonucleotide microarray. Blood, 2001, 98: 787-794

17 Voehringer D W, Hirschberg D L, Xiao J, et al. Gene microarray identification of redox and mitochondrial elements that control resistance or sensitivity to apoptosis. Proc Natl Acad Sci USA, 2000, 97 : 2680-2685

18 Yu Y, Wang L S, Shen S M, et al. Subcellular proteome analysis of camptothecin analogue NSC606985-treated acute myeloid leukemic cells. J Proteome Res, 2007, 6: 3808-3818

19 Bredemeyer A J, Townsend R R, Ley T J. Use of protease proteomics to discover granzyme B substrates. Immunol Res, 2005, 32: 143-153

20 Kruse U, Bantscheff M, Drewes G, et al. Chemical and pathway proteomics: Powerful tools for oncology drug discovery and personalized health care. Mol Cell Proteomics, 2008, 7: 1887-1901

21 Zhang J T, Liu Y. Use of comparative proteomics to identify potential resistance mechanisms in cancer treatment. Cancer Treat Rev, 2007, 33: 741-756
22 Rix U, Superti-Furga G. Target profiling of small molecules by chemical proteomics. Nat Chem Biol, 2009, 5: 616-624

23 Kohnke P L, Mulligan S P, Christopherson R I. Membrane proteomics for leukemia classification and drug target identification. Curr Opin Mol Ther, 2009, 11: 603-610

24 Riedl S J, Shi Y. Molecular mechanisms of caspase regulation during apoptosis. Nat Rev Mol Cell Biol, 2004, 5: 897-907

25 Lecellier G, Brenner C. Genomic and proteomic screening of apoptosis mitochondrial regulators for drug target discovery. Curr Med Chem, 2007, 14: 875-881

26 Cotter T G. Apoptosis and cancer: The genesis of a research field. Nat Rev Cancer, 2009, 9: 501-507

27 Chen G Q, Wang L S, Wu Y L, et al. Leukemia, an effective model for chemical biology and target therapy. Acta Pharmacol Sin, 2007, 28: $1316-1324$

28 Chen G Q, Zhang J, Zhao Q. Active compounds-based discoveries about the differentiation and apoptosis of leukemic cells. Chin Sci Bull, 2009, 54: 2759-2765

29 Chen G Q, Shi X G, Tang W, et al. Use of arsenic trioxide (As2O3) in the treatment of acute promyelocytic leukemia (APL): I. As2O3 exerts dose-dependent dual effects on APL cells. Blood, 1997, 89: 3345-3353

30 Zhang X W, Yan X J, Zhou Z R, et al. Arsenic trioxide controls the fate of the PML-RARalpha oncoprotein by directly binding PML. Science, 2010, 328: 240-243

31 Yue Q X, Cao Z W, Guan S H, et al. Proteomics characterization of the cytotoxicity mechanism of ganoderic acid D and computer-automated estimation of the possible drug target network. Mol Cell Proteomics, 2008, 7: 949-961

32 Wang X M, Yang M, Guan S H, et al. Quantitative determination of six major triterpenoids in Ganoderma lucidum and related species by high performance liquid chromatography. J Pharm Biomed Anal, 2006, 41: 838-844

33 Wang Y, He Q Y, Che C M, et al. Proteomic characterization of the cytotoxic mechanism of gold (III) porphyrin 1a, a potential anticancer drug. Proteomics, 2006, 6: 131-142

34 Wang Y, He Q Y, Sun R W, et al. GoldIII porphyrin 1a induced apoptosis by mitochondrial death pathways related to reactive oxygen species. Cancer Res, 2005, 65: 11553-11564

35 Fojo T. Multiple paths to a drug resistance phenotype: Mutations, translocations, deletions and amplification of coding genes or promoter regions, epigenetic changes and microRNAs. Drug Resist Update, 2007, 10: 59-67

36 Ouellette M, Drummelsmith J, Papadopoulou B. Leishmaniasis: Drugs in the clinic, resistance and new developments. Drug Resist Updat, 2004, 7: 257-266

37 Vergnes B, Gourbal B, Girard I, et al. A proteomics screen implicates HSP83 and a small kinetoplastid calpain-related protein in drug resistance in Leishmania donovani clinical field isolates by modulating drug-induced programmed cell death. Mol Cell Proteomics, 2007, 6: 88-101

38 Allison A C, Eugui E M. Mycophenolate mofetil and its mechanisms of action. Immunopharmacology, 2000, 47: 85-118

39 Park Y J, Ahn H J, Chang H K, et al. The RhoGDI-alpha/JNK signaling pathway plays a significant role in mycophenolic acid-induced apoptosis in an insulin-secreting cell line. Cell Signal, 2009, 21: 356-364

40 Gold L S, Slone T H, Manley N B, et al. The Carcinogenic Potency Database: Analyses of 4000 chronic animal cancer experiments published in the general literature and by the U.S. National Cancer Institute/National Toxicology Program. Environ Health Perspect, 1991, 96: $11-15$

41 Sarioglu H, Brandner S, Haberger M, et al. Analysis of 2,3,7,8tetrachlorodibenzo-p-dioxin-induced proteome changes in $5 \mathrm{~L}$ rat hepatoma cells reveals novel targets of dioxin action including the mitochondrial apoptosis regulator VDAC2. Mol Cell Proteomics, 2008, 7: 394-410

42 Chen J C, Stevens J L, Trifillis A L, et al. Renal cysteine conjugate beta-lyase-mediated toxicity studied with primary cultures of human 
proximal tubular cells. Toxicol Appl Pharmacol, 1990, 103: 463-473

43 de Graauw M, Tijdens I, Cramer R, et al. Heat shock protein 27 is the major differentially phosphorylated protein involved in renal epithelial cellular stress response and controls focal adhesion organization and apoptosis. J Biol Chem, 2005, 280: 29885-29898

44 Aridor M, Balch W E. Integration of endoplasmic reticulum signaling in health and disease. Nat Med, 1999, 5: 745-751

45 Boyce M, Bryant K F, Jousse C, et al. A selective inhibitor of eIF2alpha dephosphorylation protects cells from ER stress. Science, 2005, 307: 935-939

46 Boyce M, Py B F, Ryazanov A G, et al. A pharmacoproteomic approach implicates eukaryotic elongation factor 2 kinase in ER stress-induced cell death. Cell Death Differ, 2008, 15: 589-599

47 Song M G, Gao S M, Du K M, et al. Nanomolar concentration of NSC606985, a camptothecin analog, induces leukemic-cell apoptosis through protein kinase Cdelta-dependent mechanisms. Blood, 2005, 105: 3714-3721

48 Liu W, Zhu Y S, Guo M, et al. Therapeutic efficacy of NSC606985, a novel camptothecin analog, in a mouse model of acute promyelocytic leukemia. Leuk Res, 2007, 31: 1565-1574

49 Shen S M, Yu Y, Wu Y L, et al. Down-regulation of ANP32B, a novel substrate of caspase-3, enhances caspase-3 activation and apoptosis induction in myeloid leukemic cells. Carcinogenesis, 2009, 31: 419-426

50 Yuan T T, Huang Y, Zhou C X, et al. Nuclear translocation of dihydrofolate reductase is not a pre-requisite for DNA damage induced apoptosis. Apoptosis, 2009, 14: 699-710

51 Gao F H, Wu Y L, Zhao M, et al. Protein kinase C-delta mediates down-regulation of heterogeneous nuclear ribonucleoprotein $\mathrm{K}$ protein: involvement in apoptosis induction. Exp Cell Res, 2009, 315: 3250-3258

52 Zheng Y, Wang L S, Xia L, et al. NDRG1 is down-regulated in the early apoptotic event induced by camptothecin analogs: The potential role in proteolytic activation of PKC delta and apoptosis. Proteomics, 2009, 9: 2064-2075

53 Vazquez A, Bond E E, Levine A J, et al. The genetics of the p53 pathway, apoptosis and cancer therapy. Nat Rev Drug Discov, 2008, 7: 979-987

54 Lessene G, Czabotar P E, Colman P M. Bcl-2 family antagonists for cancer therapy. Nat Rev Drug Discov, 2008, 7: 989-1000

55 Kerr L E, Birse-Archbold J L, Short D M, et al. Nucleophosmin is a novel Bax chaperone that regulates apoptotic cell death. Oncogene, 2007, 26: 2554-2562

56 Docquier F, Farrar D, D'Arcy V, et al. Heightened expression of CTCF in breast cancer cells is associated with resistance to apoptosis. Cancer Res, 2005, 65: 5112-5122

57 Hwang S I, Lundgren D H, Mayya V, et al. Systematic characterization of nuclear proteome during apoptosis: A quantitative proteomic study by differential extraction and stable isotope labeling. Mol Cell Proteomics, 2006, 5: 1131-1145

58 Danial N N, Gramm C F, Scorrano L, et al. BAD and glucokinase reside in a mitochondrial complex that integrates glycolysis and apoptosis. Nature, 2003, 424: 952-956

59 Liu X, Pan Z, Zhang L, et al. JAB1 accelerates mitochondrial apoptosis by interaction with proapoptotic BclGs. Cell Signal, 2008, 20: 230-240

60 Cimmino A, Capasso R, Muller F, et al. Protein isoaspartate methyltransferase prevents apoptosis induced by oxidative stress in endothelial cells: Role of Bcl-X1 deamidation and methylation. PLoS ONE, 2008, 3: e3258

61 Shiio Y, Suh K S, Lee H, et al. Quantitative proteomic analysis of myc-induced apoptosis: A direct role for Myc induction of the mitochondrial chloride ion channel, mtCLIC/CLIC4. J Biol Chem, 2006, 281: 2750-2756

62 Jenkins L M, Mazur S J, Rossi M, et al. Quantitative proteomics analysis of the effects of ionizing radiation in wild type and p53 K317R knock-in mouse thymocytes. Mol Cell Proteomics, 2008, 7: 716-727

63 Tang Y, Zhao W, Chen Y, et al. Acetylation is indispensable for p53 activation. Cell, 2008, 133: 612-626

64 Gu S, Liu Z, Pan S, et al. Global investigation of p53-induced apoptosis through quantitative proteomic profiling using comparative amino acid-coded tagging. Mol Cell Proteomics, 2004, 3: 998-1008

65 Johnson M D, Yu L R, Conrads T P, et al. Proteome analysis of DNA damage-induced neuronal death using high throughput mass spectrometry. J Biol Chem, 2004, 279: 26685-26697

66 Rahman-Roblick R, Roblick U J, Hellman U, et al. p53 targets identified by protein expression profiling. Proc Natl Acad Sci USA, 2007, 104: 5401-5406

67 Rahman-Roblick R, Hellman U, Becker S, et al. Proteomic identification of p53-dependent protein phosphorylation. Oncogene, 2008, 27: 4854-4859

68 Orre L M, Pernemalm M, Lengqvist J, et al. Up-regulation, modification, and translocation of S100A6 induced by exposure to ionizing radiation revealed by proteomics profiling. Mol Cell Proteomics, 2007, 6: 2122-2131

69 Webb P R, Wang K Q, Scheel-Toellner D, et al. Regulation of neutrophil apoptosis: A role for protein kinase $\mathrm{C}$ and phosphatidylinositol-3-kinase. Apoptosis, 2000, 5: 451-458

70 Galietta A, Gunby R H, Redaelli S, et al. NPM/ALK binds and phosphorylates the RNA/DNA-binding protein PSF in anaplastic large-cell lymphoma. Blood, 2007, 110: 2600-2609

71 McDonald P C, Oloumi A, Mills J, et al. Rictor and integrin-linked kinase interact and regulate Akt phosphorylation and cancer cell survival. Cancer Res, 2008, 68: 1618-1624

72 Kang S, Dong S, Gu T L, et al. FGFR3 activates RSK2 to mediate hematopoietic transformation through tyrosine phosphorylation of RSK2 and activation of the MEK/ERK pathway. Cancer Cell, 2007, 12: 201-214

73 Baccarini M. An old kinase on a new path: Raf and apoptosis. Cell Death Differ, 2002, 9: 783-785

74 Huser M, Luckett J, Chiloeches A, et al. MEK kinase activity is not necessary for Raf-1 function. EMBO J, 2001, 20: 1940-1951

75 O'Neill E, Rushworth L, Baccarini M, et al. Role of the kinase MST2 in suppression of apoptosis by the proto-oncogene product Raf-1. Science, 2004, 306: 2267-2270

76 Matallanas D, Romano D, Yee K, et al. RASSF1A elicits apoptosis through an MST2 pathway directing proapoptotic transcription by the p73 tumor suppressor protein. Mol Cell, 2007, 27: 962-975

77 Timmer J C, Salvesen G S. Caspase substrates. Cell Death Differ, 2007, 14: 66-72

78 Lu Y, Peng Z G, Yuan T T, et al. Multi-sites cleavage of leukemogenic AML1-ETO fusion protein by caspase-3 and its contribution to increased apoptotic sensitivity. Leukemia, 2008, 22: 378-386

79 Stennicke H R, Renatus M, Meldal M, et al. Internally quenched fluorescent peptide substrates disclose the subsite preferences of human caspases 1, 3, 6, 7 and 8. Biochem J, 2000, 350: 563-568

80 Lakhani S A, Masud A, Kuida K, et al. Caspases 3 and 7: Key mediators of mitochondrial events of apoptosis. Science, 2006, 311: 847-851

81 Walsh J G, Cullen S P, Sheridan C, et al. Executioner caspase-3 and caspase-7 are functionally distinct proteases. Proc Natl Acad Sci USA, 2008, 105: 12815-12819

82 Stroh C, Schulze-Osthoff K. Death by a thousand cuts: An ever increasing list of caspase substrates. Cell Death Differ, 1998, 5: 9971000

83 Luthi A U, Martin S J. The CASBAH: A searchable database of caspase substrates. Cell Death Differ, 2007, 14: 641-650

84 Demon D, Van Damme P, Berghe T V, et al. Caspase substrates: Easily caught in deep waters? Trends Biotechnol, 2009, 27: 680-688

85 Wee L J, Tan T W, Ranganathan S. CASVM: Web server for SVM-based prediction of caspase substrates cleavage sites. Bioinformatics, 2007, 23: 3241-3243

86 Dix M M, Simon G M, Cravatt B F. Global mapping of the topography and magnitude of proteolytic events in apoptosis. Cell, 2008, 134: 679-691

87 Mahrus S, Trinidad J C, Barkan D T, et al. Global sequencing of proteolytic cleavage sites in apoptosis by specific labeling of protein 
N termini. Cell, 2008, 134: 866-876

88 Enoksson M, Li J, Ivancic M M, et al. Identification of proteolytic cleavage sites by quantitative proteomics. J Proteome Res, 2007, 6: 2850-2858

89 Van Damme P, Martens L, Van Damme J, et al. Caspase-specific and nonspecific in vivo protein processing during Fas-induced apoptosis. Nat Methods, 2005, 2: 771-777

90 Impens F, Van Damme P, Demol H, et al. Mechanistic insight into taxol-induced cell death. Oncogene, 2008, 27: 4580-4591

91 Bredemeyer A J, Lewis R M, Malone J P, et al. A proteomic approach for the discovery of protease substrates. Proc Natl Acad Sci USA, 2004, 101: 11785-11790
92 Adrain C, Murphy B M, Martin S J. Molecular ordering of the caspase activation cascade initiated by the cytotoxic $\mathrm{T}$ lymphocyte/natural killer (CTL/NK) protease granzyme B. J Biol Chem, 2005, 280: 4663-4673

93 Saelens X, Festjens N, Vande Walle L, et al. Toxic proteins released from mitochondria in cell death. Oncogene, 2004, 23: 2861-2874

94 Hegde R, Srinivasula S M, Zhang Z, et al. Identification of Omi/HtrA2 as a mitochondrial apoptotic serine protease that disrupts inhibitor of apoptosis protein-caspase interaction. J Biol Chem, 2002, 277: 432-438

95 Vande Walle L, Van Damme P, Lamkanfi M, et al. Proteome-wide identification of HtrA2/Omi substrates. J Proteome Res, 2007, 6: 1006-1015

Open Access This article is distributed under the terms of the Creative Commons Attribution License which permits any use, distribution, and reproduction in any medium, provided the original author(s) and source are credited. 